\title{
Roadside Vertical Axis Wind Turbine (VAWT): An Effective Evolutionary Design for Australian Highway Commuters with Minimum Dynamic Stall
}

\author{
Assies Ahmad1, Adil Loya', Muhammad Ali³, Ammar Iqbal', Faiq Masood Baig1, \\ Abdul Manan Afzal4 \\ ${ }^{1}$ Department of Engineering, University of Technology, Sydney, Australia \\ ${ }^{2}$ Department of Mechatronics Engineering, PAF Karachi Institute of Economics and Technology, Karachi, Pakistan \\ ${ }^{3}$ Department of Mechanical Engineering, COMSATS University Islamabad, Sahiwal Campus, Sahiwal, Pakistan \\ ${ }^{4}$ Department of Automotive Engineering, University of Engineering and Technology, Lahore Campus, Lahore, Pakistan \\ Email: loya_adil@yahoo.com
}

How to cite this paper: Ahmad, A., Loya, A., Ali, M., Iqbal, A., Baig, F.M. and Afzal. A.M. (2020) Roadside Vertical Axis Wind Turbine (VAWT): An Effective Evolutionary Design for Australian Highway Commuters with Minimum Dynamic Stall. Engineering, 12, 601-616.

https://doi.org/10.4236/eng.2020.129042

Received: June 24, 2020

Accepted: September 4, 2020

Published: September 7, 2020

Copyright $\odot 2020$ by author(s) and Scientific Research Publishing Inc. This work is licensed under the Creative Commons Attribution International License (CC BY 4.0).

http://creativecommons.org/licenses/by/4.0/

cc) (i) Open Access

\begin{abstract}
There are multiple approaches of design for Vertical Axis Wind Turbines (VAWT) that have been studied by engineers and leaps have been made in high performing innovations. By harnessing the energy from these wind turbines, the problem of roadside lights shortage can be solved. This can help to prevent the accidents while providing clean energy. The importance of coastal areas like Australian beaches regarding wind turbines cannot be neglected as a higher number of people like to live near coastal vicinity. Also, most of the freeways in Australia expand across the sea. In this paper, one such design has been analyzed to implement across the highways. But still with many advancements in technology, an immense gap is present in the research of implementation of VAWTs. The design discussed in the current study is a VAWT which can be installed on the side of the highway roads to provide clean and cheap energy for illuminating the roads. Computational Fluid Dynamics (CFD) was conducted on the blades of the turbine to analyze its performance under operating conditions. Furthermore, the paper elaborates the generation of drag and lift on the blades of the turbine. A wind speed of 60 $\mathrm{km} / \mathrm{h}$ just produced $6.1 \mathrm{~N}$ force on the turbine blades as a result of drag. The cost analysis showed the cheap production of such mechanism that can provide longer service when installed.
\end{abstract}

\section{Keywords}

Aerodynamics, Computational Fluid Dynamics, Vertical Axis Wind Turbine, 
Finite Elemental Analysis, Simulations

\section{Introduction}

For past few decades, there has been a rise in the factors that are damaging the earth. Their extent is reached to all the natural phenomenon and ecosystems. With this rate, the upcoming generations will have to suffer the most from it. Energy is one of the main resources that support the lifestyle of the humans in current era but almost $80 \%$ of energy produced today is generated from the fossil fuels [1]. With the ever-increasing industry the use of natural resources has increased tenfold. This has caused them to deplete at very high rates. It is commonly assumed that the complete depletion of these finite resources will throw the world out of balance.

Currently, the world is in dire need of the renewable energy sources due to the adverse effects of the conventional ones. Also, many researches have proven that the renewable energy sources are way cheaper than the conventional methods in terms of production. There are many sources around the globe that can generate renewable energy. Many researches have shown that earth has a great capability of wind energy especially along the coastlines [2]. The concept of generating energy through wind was first introduced in 1931 which led to the invention of Vertical Axis Wind Turbine (VAWT). Although Horizontal Axis Wind Turbines (HAWT) had already caught researchers' attention [1]. The general working of a wind turbine is not very complex. The main parts of the turbine are its returning blades, which are attached to the main rotor part with the help of radial arms.

Recently, the researchers have turned their attention towards VAWT. Even though this turbine is very cost effective and it is easier to manufacture, however, there is a big research gap left by the researchers on VAWT due to their unfettered working on HAWT [3]. Many researchers have provided a lot of new innovative ideas to use the wind energy effectively, but most of them couldn't be implemented. It is the need of time that this industry elaborates and finds the solutions of the fundamental aspects that question the credibility of (Wind energy efficiency evaluation procedure).

One of the best places to gain the most from the wind energy is Australia as most of its population lives along the coastal areas. Such areas are ideally used to harness the wind energy as they have violent winds [4]. Due to the country's size, supply of electricity to all the people is a really big challenge, especially when it comes to highways. The lights on highways are very important as there have been a lot of road accidents due to its absence on the roads under various circumstances [5].

Another reason for the accidents is the extra fatigue drivers have to face during travel. Drivers get under the influence of fatigue mostly at night time and near the regional areas. It is thereby required that the lights on the roads must be given equal importance like other programs of road safety such as limiting the 
hours of work, taking time to rest, etc. [5]. Road lights are very important and they can be provided energy using renewable sources. A successful implementation in this regard are the lights that use solar energy. This method has opened gateways for renewable energy use in this regard. Researchers are now looking forward to refine these ideas.

Wind energy is now being used on larger scales and now contributes $3.8 \%$ to the total energy of the world [6]. The two commonly used wind turbines are:

1) HAWT (Horizontal Axis Wind Turbine)

2) VAWT (Vertical Axis Wind Turbine)

A comparison between the two turbines has been shown in Figure 1 [7]. The main difference between VAWT and HAWT is the position of the main shaft. The shaft is perpendicular to the wind direction in VAWT while it is parallel in case of HAWT. The usage of HAWT is very vast in industrial applications. The researcher's lack of interest in VAWT has caused a great gap in its innovation and practical applications. This gap has led to the failure of the VAWT turbine designs in recent years and this has also created hesitation among the researchers. One of the main reasons for these failures is that there are not enough studies conducted on the CFD analysis of these turbines [8]. To achieve the complete aerodynamical analysis multiple diagnostic approaches are applied including numerical, computational and experimental setups. This helps in authenticating the results and efficiency [9]. With the help of the critical analysis done on these diagnostics, it was established that DMST (Double Multiple Steam-tube Theory) is a very realistic technique that can provide result-oriented analysis. This theory is classified as the most reliable in terms of precision of results [10].

Through DMST it was established that the factors that influence the pitch of airfoil design are fluid wake in reverse between the blades of the turbine, peak flow and drag due to downwind [11]. Using combination technique of eyebrow, azimuthal angle and factors of wing aspect ratio, the thrust efficiency of the airfoil can be enhanced. The power efficiency is calculated via numerical computations

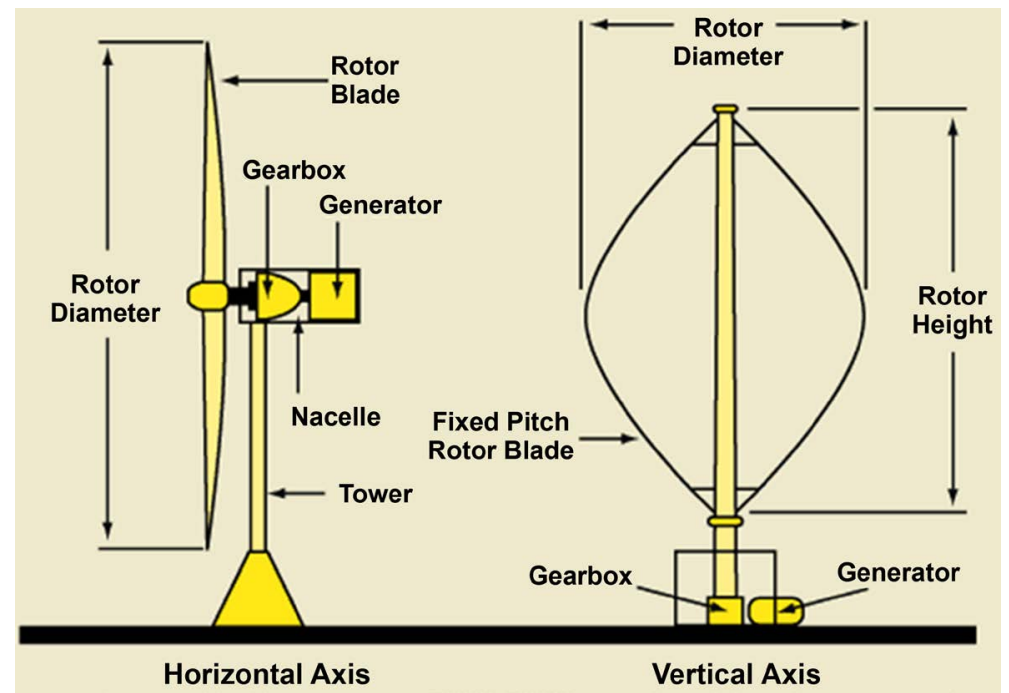

Figure 1. Horizontal \& Vertical axis wind turbine [7]. 
and the evaluation of the drag forces takes place by putting airfoil inside actuator stream [12].

This method of analysis is only limited to the turbines of smaller scales which makes it more ideal for this study. The wind peak and average wind speeds of various coastal areas in Australia were taken into account for the boundary condition of the study [13]. This study will provide insight of the critical factors that have led to the lesser use of DMST in literature.

\section{Literature Review}

The use of wind turbine goes back to $7^{\text {th }}$ century in the region of middle east. They had a very small efficiency and operated on horizontal axis. Several centuries later the Dutch developed windmills that were used to pump out the water. US used wind turbines to irrigate the lands in $19^{\text {th }}$ century. The first wind turbine to generate electricity was developed in the early 90 s [14].

\subsection{Comparison between HAWT and VAWT}

The benefits of VAWT are more than that of HAWT. For most of the VAWT designs, the wind traction mechanism is not required. HAWTs have a lesser efficiency in the areas where the turbulence of the winds is very high. The reason is that a high amount of fatigue is caused by these winds. As compared to that the performance of VAWT is quite higher in all such conditions [15]. The less amount of fatigue is generated in VAWT due to its assembly consists of smaller gearboxes. The design of these turbines is very simple and gives the accessibility to the gearboxes from the ground. While in HAWT an elevated platform is required to access the gearboxes in order to maintain them [16].

By carefully designing the VAWT the obtained output can be increased tenfold as compared to HAWT [17]. In certain assemblies of VAWT screws are used and can be transported to other locations, while the long blades of HAWT can't be broken down and much more difficult to transport [18].

The performance of VAWT is greatly affected by the dynamic stall due to the changing wind angle of attack [19]. The airfoils are affected greatly by the stall as the variation of windspeed causes a heavy turbulence around them. On one side the varying stall onset is higher rather than on the other side [20]. This causes delay on the beginning movement of the blades and the reattachment of the airflow is also affected. The structural integrity of the turbine comes to a risk due to the generation of the vortexes at the back of the blades. These can cause a great load of vibrations creating higher fatigues as well as the loads [21].

\subsection{Aerodynamic Forces}

The aerodynamic forces acting on the blades cause the rotation of turbine. These forces can be resolved into just drag and lift [22]. The forces of lift help in making the blades lighter and the drag is responsible for the movement and they act on the airfoils at the same time [23]. The parameters to determine how much 
drag and lift will act on the blades are same as the other foils. These parameters include blades' surface area, as well as the wind speed [24]. To calculate the drag and lift the drag coefficient and the lift coefficients are used respectively.

\subsection{CFD Working}

CFD analysis solves the problems related to aerodynamics as by using mathematical models and equations. Steps that are included in a CFD analysis are as under:

- Entering the data in computer.

- Making a mathematical model.

- Constructing the mathematical model in form of computer algorithms.

- Calculations using computer.

- Acquiring the data.

- Analysis after the calculations.

The wind turbines are composed of mechanical components including shafts, bearings, gear boxes, airfoils and gears. Lubrication is applied to the gear boxes and the bearings. According to the requirements, these components can be replaced while performing maintenance [25]. It is more costly to repair big VAWTs through run to failure maintenance. In order to prevent that remote monitoring is used which provides monitoring of the vibrations, heat and other issues in the turbine in real time [26].

\section{Methodology}

Best concept for the turbine was chosen by idealizing multiple models and then selecting the best one among them. The selected model was designed in SolidWorks. In order to choose the material for the analysis, its part was selected on Cambridge Engineering Selector (CES) software. For the investigation of fluid dynamics and strength, various simulations were conducted. Cost analysis of the model was also performed in order to find out the feasibility of the design.

\subsection{Design Phase}

The design of the turbine is shown in Figure 2. In the first step the parts were generated and the appropriate part material was selected for the design. After selecting the material design set optimization was carried out. The drawings of the part were also drafted.

Before designing the turbine, a few of things were kept in consideration. First one was to make sure that the design could fit in the road divider. At the lower part of the turbine the speed of wind is lower due to the ground friction [27]. It was designed with 3 blades which are shown in yellow color in Figure 2. By using the tri-plates at the top and the bottom all three blades were provided with the support. Another purpose that tri-plates serve was keeping the blades at a steady angle from each other during the rotation. The complete structure was supported via a middle rotating rod. It was made of aluminum and its main 


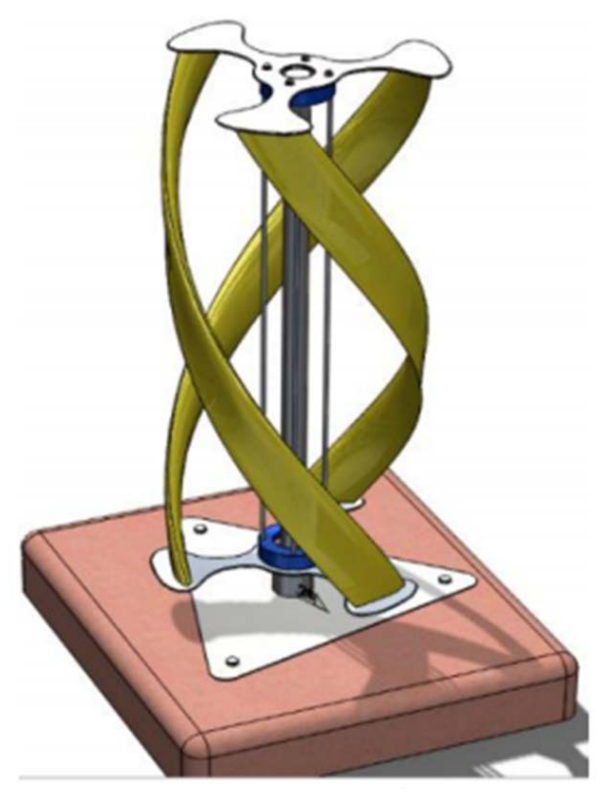

Figure 2. CAD design in solidworks.

purpose was to keep the turbine at vertical angle. The rod was welded on a triangular plate at the bottom and the plate was fixed in the base by $3 \mathrm{M} 16$ bolts.

A slab was designed to be used as the base of the turbine. Its main purpose was to anchor the turbine strongly to be placed in the road divider without sinking. The turbine was also attached with the bearing housings at the bottom and the top. These bearings were made of Acrylonitrile butadiene styrene (ABS) and they were attached with the help of bolts on the tri-plates. The spacer operated inside them.

The inner housings were connected to the bearings. In order to provide extra strength to the spacers, 4 members were also installed and connected at top and bottom ends. These members were made of aluminum and were inserted in specific holes around the spacers. The height of the model was $1530 \mathrm{~mm}$ and the blades weighed $3.4 \mathrm{~kg}$ [28].

\subsubsection{Turbine Dimensions}

The height of the turbine was kept smaller due to its location of the operation. At $1.53 \mathrm{~m}$ the turbine would stay stable and will also not obstruct driver's view while rotating in the center of the highway. The turbine was specifically designed to install on a road divider on the highway. So, in that context, its width had to be kept lesser so that it would not overhang on the divider. The turbine was kept at $60 \mathrm{~cm}$ of width which was enough to fit on the road divider on the highway. The total width and total length of turbine were $600 \mathrm{~mm}$ and $1529.08 \mathrm{~mm}$ as shown in Figure 3.

All 3 blades of the turbine in this model were given a curved design around the axis. The separation among the 3 blades was 120 degrees. Interacting wind hit the airfoils in a helical pattern. The wind gradually moved down at one end of the blades and left smoothly on the other. This flow of the air through the 


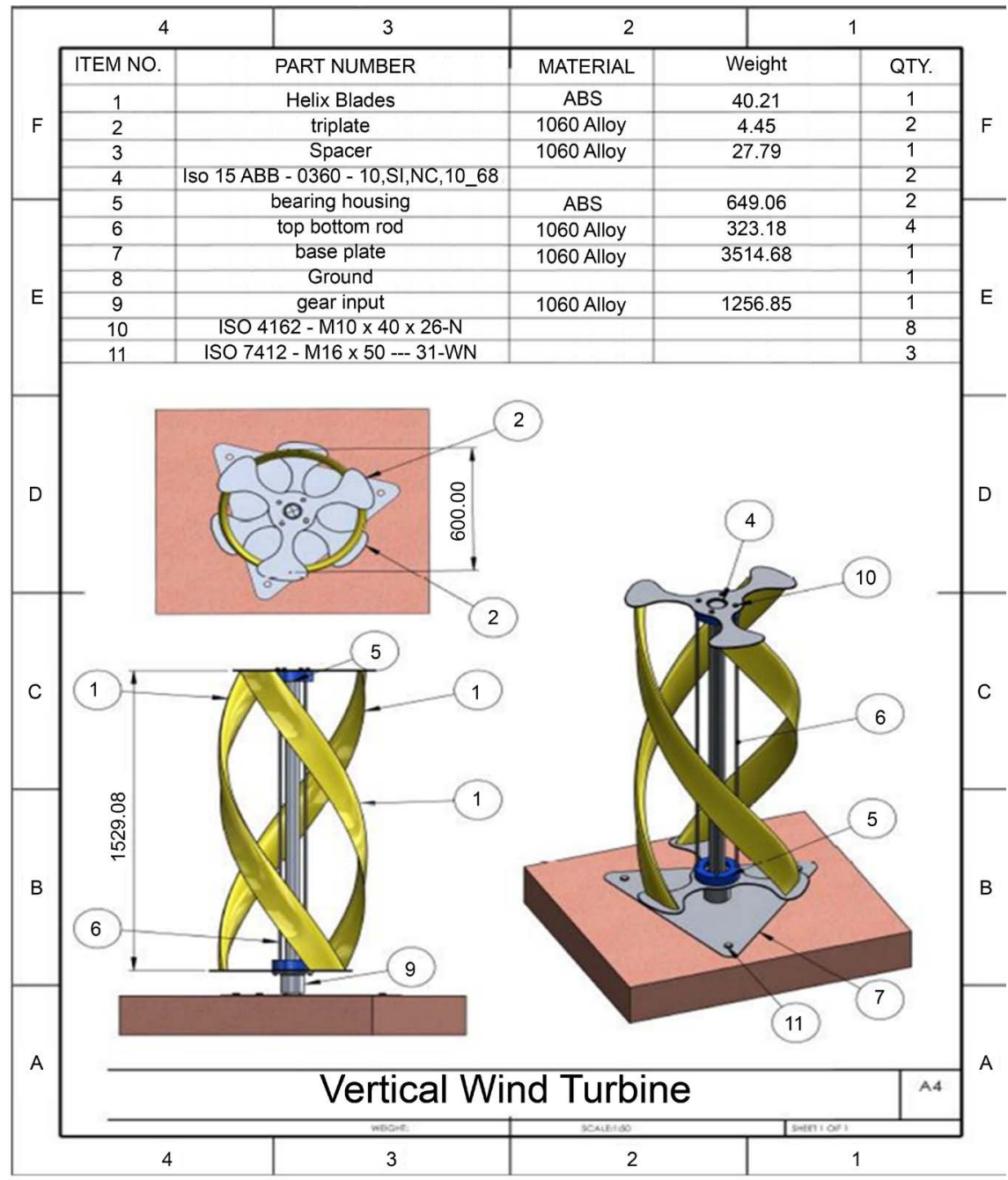

Figure 3. CAD design with basic dimension and Bill of Material from solidworks.

wind depicted the meshing of 2 helical gears; gradual, quiet and smooth i.e. when one blade was forced by the wind to move the next one gradually took its place and the cycle was continued.

\subsubsection{Smooth Airfoil Curve}

To improve the aerodynamics of the turbine its airfoils were designed similar to an airplane. The design had much more capability to generate lift force on the blades while reducing the drag acting on them. In this way, the blades were able to harness maximum wind energy while facing lesser drag resistance. The blades had more rounded surface on outer edges as compared to the inner edges. This created a difference of pressure between inner and outer edges which created lift towards the outside of the blades.

\subsubsection{Good Clearance}

It is commonly known that stronger winds will produce more energy but the effects they produce can sometimes become devastating and it gets even difficult for the wind turbines to handle them. There are certain regions in Australia that 
undergo heavy winds and storms and the wind speed can reach up to $200 \mathrm{~km} / \mathrm{h}$. At such high speeds the structures in the area can become vulnerable including wind turbines.

Turbines stop moving at this point and can be pushed by the winds if the blockage of air is maximum. This model was specifically designed with wider blades space to somewhat overcome this issue. Structures like billboards that are more likely to be affected by this phenomenon. So, the new designs include perforations to allow the air passage.

\subsubsection{Enough Height}

According to the study, by increasing the height of the blades twice in size the production of energy will also increase. But this may not be the case for all turbines. The regions having lesser wind speeds can show the opposite results. In these areas, a smaller turbine is recommended as it can produce more energy than a taller one. The selected for the design is preferable to be used in the areas where lower wind speeds are produced.

In case of the wind turbines that are most commonly used (horizontal axis), the height of the tower is very important in determining their performance. The ones having higher towers will produce more energy.

For a taller design, a larger base would be required for this study to make the design more stable and eventually more space would be required. The whole purpose of the design was to keep it compact so that it could produce maximum energy in the given parameters. A smaller design would lead to a performance deficient model.

\subsubsection{Mechanical Friction Reduction}

The two ball bearings installed at the top and the bottom of the turbine ensured lesser friction during the operations. The ball bearings made the design more efficient.

\subsection{Material Selection}

The material for the turbine was selected with the help of CES. This is a software that have predefined material libraries in it. The parameters of the material are input to the software then it compares those parameters to the material libraries and selects the materials based on the given parameters. Every material parameter group searched by the software is called stage. When the final material is chosen that means it has passed through all the stages. For this study, the blades and the spacers were the 2 most important components of the design. The blades had to catch the incoming wind and with the force exerted by the air the spacer had to rotate.

\subsubsection{Material for Blade}

It was most crucial for the turbine's performance to choose a light, corrosion resistant and cheap material for the blades. In this regard, Aluminum was chosen. This material was not only light in weight but also had high recyclability. The 
density of this material is very low. So, each blade weighed 40 ounces and 120 ounces or $3.4 \mathrm{~kg}$ combined.

\subsubsection{Material for Spacer}

The spacer is another important component in the design. The material for spacer had to be strong, light-weight and corrosion resistant. CES recommended three materials for this purpose which were stainless steel, alloys of aluminum and carbon steel. The most expensive material among them was stainless steel while the carbon steel had higher tendencies of rusting due to water and moisture. Based on the data both of the materials were not suitable for the operations. Aluminum 6061 was chosen due to its resistivity from the corrosion and strength [28].

\subsection{Simulation Phase}

There were 3 different analysis performed on the turbine design to find out the results of forces acting on the structures. These analyses included equivalent stresses, lift and drag forces on the turbine blades. A cost analysis was also performed.

\section{Stress Analysis (Static Test on Spacer)}

The simulations were run on spacer in order to find out the stresses generating in the structure due to the wind hitting the blades of the turbine.

\subsection{Pre-Processing}

The FEA was used to run the simulations on the spacer. The first step was to create a mesh on the spacer. The size of the element was 0.284415 inch while the element numbers and the node numbers were 60002 and 100605 respectively. An aspect ratio of 13.906 was given to the mesh.

The material of the spacer was configured in SolidWorks. The spacer was fixed at the bottom end while a push force of $300 \mathrm{~N}$ was applied at the top ends. The configuration of the fixer is shown in Figure 4.

FEA was carried out for evaluating spacers stiffness during an impact with the winds. As this impact exerts maximum pressure on the upper part of the structure rather than the lower part where the turbine was anchored. The simulations were conducted using a fine mesh. This mesh is shown in Figure 5. Fine meshes always consist of a greater number of nodes which helps in generating more accurate results.

\section{Results}

\subsection{Von Mises Stresses and Displacement}

In Figure 6 and Figure 7 von mises stresses and deformation displacements results are represented accordingly.

- The maximum von mises or equivalent stress which was $1.884 \mathrm{e}+07$, generated at the bottom of the spacer where it was fixed with the base while the minimum stress which was $9.003 e+04$ generated at the top. 


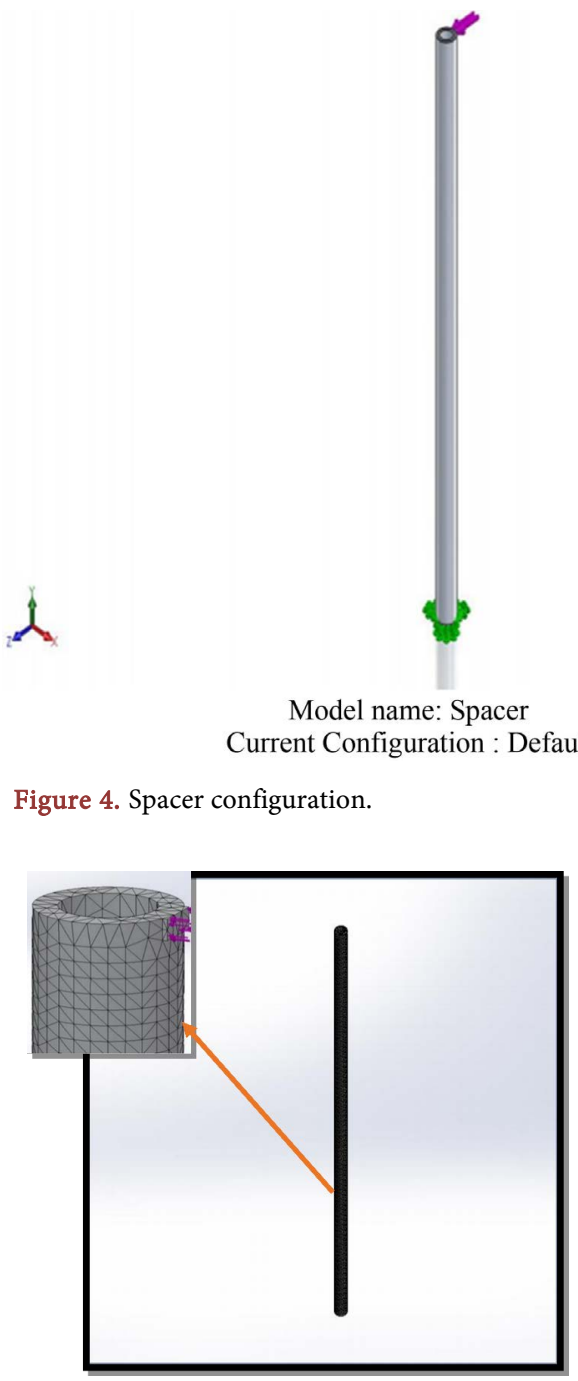

Figure 5. Meshing of the spacer.

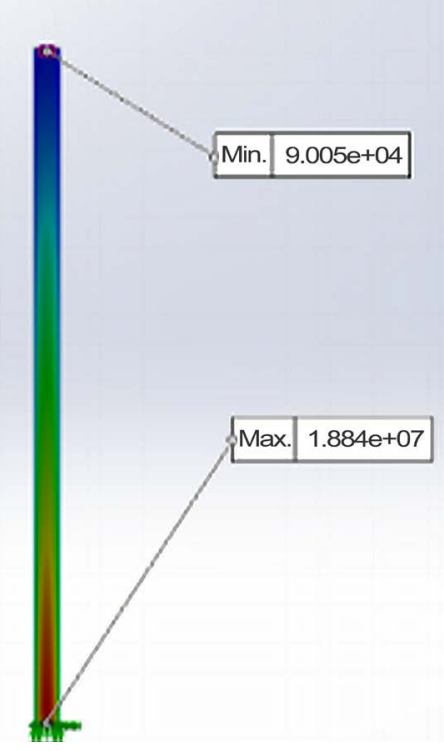

von Mises (N/m^2)

Figure 6. Von Mises stresses. 


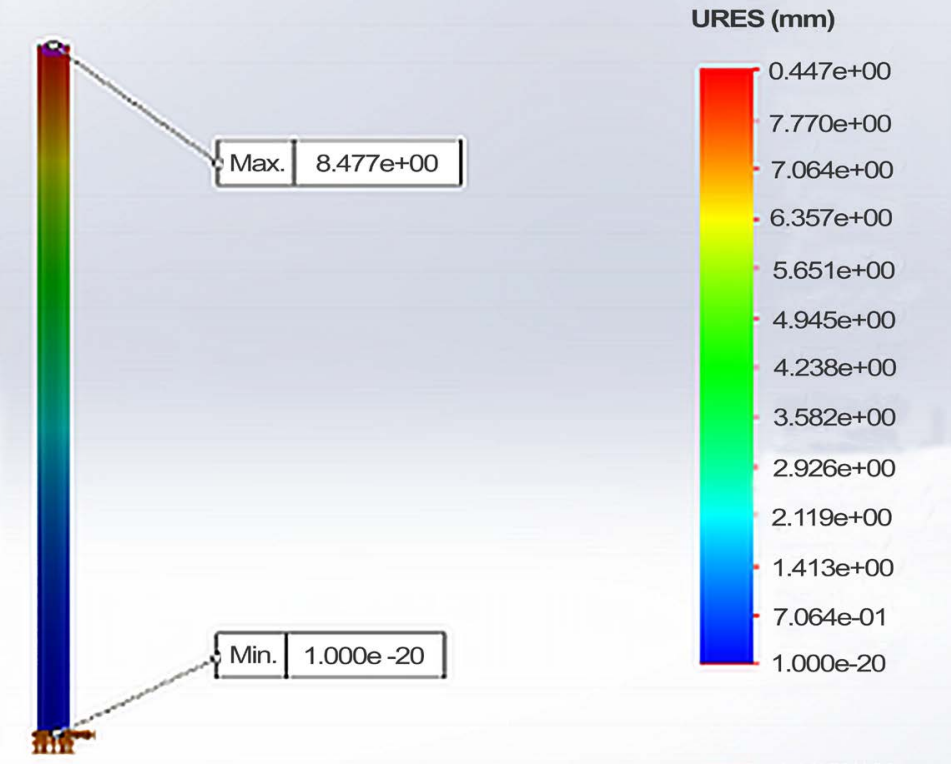

Figure 7. Displacement.

- The yield strength of the spacer was $2.757 \mathrm{e}+07$. The maximum stresses generated in the member did not exceed the specified yield strength, which represented that the model will not fail during loading due to the stresses.

- As assumed the maximum deflection occurred at the top of the spacer which was $8.477 \mathrm{e}+00$ URES $(\mathrm{mm})$.

\subsection{Computational Fluid Dynamics (CFD)}

\subsubsection{Turbine Fluid Dynamic Characteristics}

With the help of the SolidWorks flow analysis the stall on the blades of the turbine was calculated via CFD analysis. The speed of the wind was kept $60 \mathrm{~km} / \mathrm{h}$ while the temperature was kept at average room temperature. The simulations were run with the mesh setting 5 i.e. the size of the element was kept at $5 \mathrm{~mm}$.

The drag coefficient for this simulation was 0.1958 . This determined the intensity of the drag on the blades. This was a very small value. In ideal cases, there is no dynamic stall and the drag coefficient is 0 . Only $6.1 \mathrm{~N}$ force was generated by this drag which was also very small value. The iterations were run until $100 \%$ converged solution was achieved as shown in Figure 8 and Figure 9 for drag coefficient and lift coefficient.

Figure 8 showed the graph of drag coefficient. It could be seen in the graph that the value of the drag coefficient was higher in the beginning of the iterations and then slowly it monotonically decayed and became constant as the iterations reached the converged solution. The results in the graph were positive which showed that the drag was never 0 .

Figure 9 showed the graph of lift coefficient. The pattern of the lift was different as compared to the drag. The lift converged at 0.007474032 after this it began to decay monotonically. Lift coefficient was also positive showing that there will always be lift acting on the turbine. 


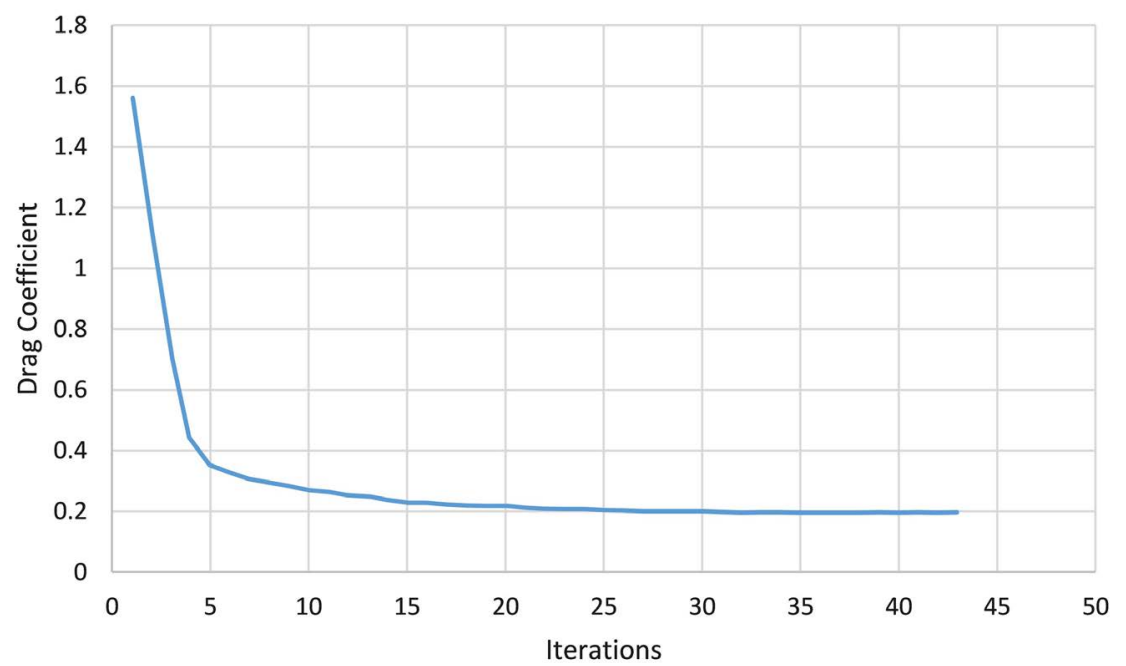

Figure 8. Drag coefficient graph.

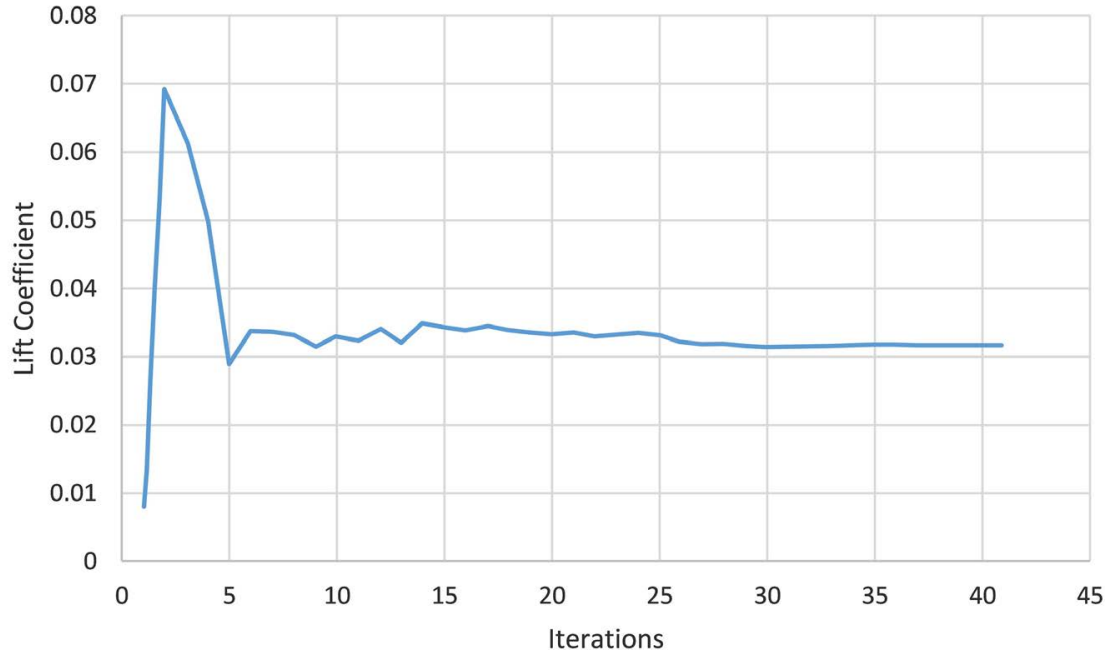

Figure 9. Lift coefficient graph.

\subsubsection{Flow Trajectories}

The simulations showed that smaller number of vortexes were generated. This was due to the reason that the helical shape of the blades deflected the wind very well. Lesser vortexes also showed that smaller stall was generated. The flow of the air around the turbine blades were indicated in Figure 10.

In this simulation, just like other simulations such a Finite Element Analysis, green and blue colors of lines and arrow heads indicate that the surface pressures are allowable. Colors of red, yellow and orange indicate that there is part or surfaces are under extreme pressures or stress. This demonstrated that the turbine was under the pressure zones prescribed according to the allowable yield strength of the material showing capability of withstanding the stresses.

\subsection{Cost Analysis}

This analysis was also carried out with the help of SolidWorks. The excerpt report of the cost of various components in the assembly is as under: 


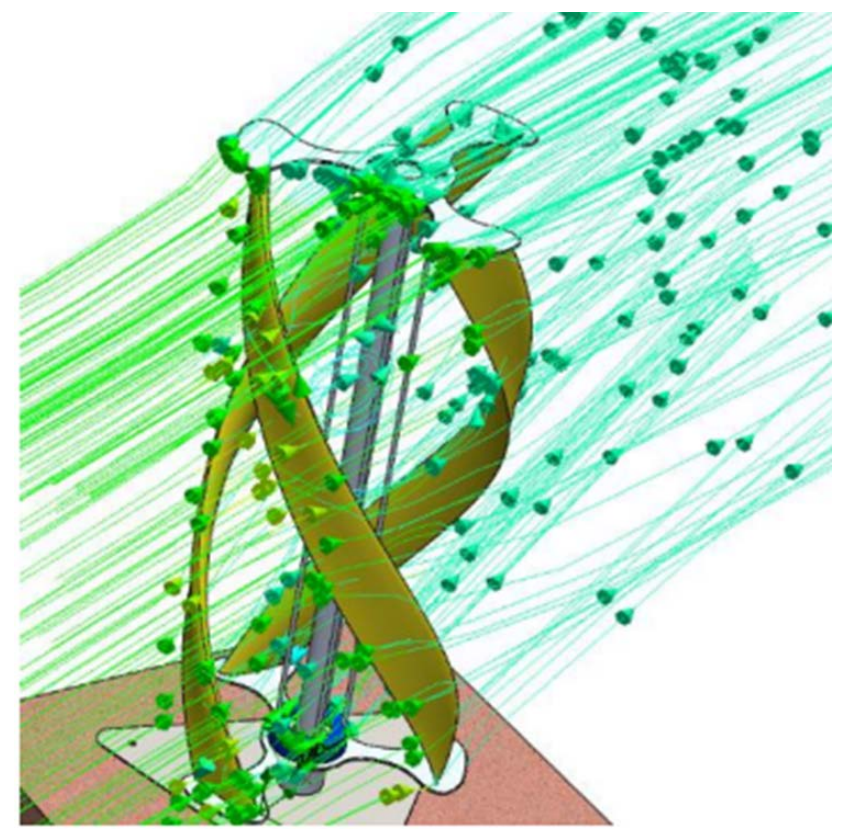

Figure 10. Flow trajectory.

\section{Component Cost Impact}

The costing of the components had been shown in Table 1 and Table 2. As the tables showed that the helix blades had high costs. The least expensive was the bearing housings as they were made of ABS plastics.

Some of the components were purchased from the market like bolts etc. Without the cost of labor, the total cost of the design would be USD 121.65. It must be kept in mind if these components were manufactured, they would have a higher cost than this. Achieving tolerances and precision of design would also result in high machinability.

\section{Conclusions}

The advantages of VAWT are larger than that of the HAWT regarding the performance parameters. The helical and spiral structures in VAWTs do not require special mechanisms to compensate the air traction. This leads to less complex design. The material of the blades must be cheap, light weight and corrosion resistant. Remote monitoring can be done in order to detect the issues occurring in the turbines in real time. The results in the studies showed that the design performed adequately under various analyses. The maximum stress was well under the yield strength that showed that the turbine had a strong structure and it will not fail. The drag forces were lower and generated lesser resistive forces. This became more evident when the flow trajectory showed fewer vortexes. The lift was also generated as the blades were designed like the airfoils of an airplane. Lastly, the cost of the design was not very high as some of the components used inside it could be directly purchased from the market.

This study revealed that a vertical wind turbine can perform well if the appropriate geometry is used. Yet there are still a lot of gaps to be fulfilled regarding 
Table 1. Blade finances.

\begin{tabular}{|c|c|c|c|c|}
\hline Component & Configuration & Material & Manufacturing Cost & Total \\
\hline & & (USD/Assembly) & (USD/Assembly) & (USD/Assembly) \\
\hline Helix Blades & Default & 20.89 & 12.98 & 33.87 \\
\hline Gear input & Default & 10.00 & 2.69 & 12.69 \\
\hline Tri-plate & Default & 12.45 & 1.78 & 14.23 \\
\hline Base Plate & Default & 17.78 & 3.12 & 20.9 \\
\hline Bearing & Default & 4.00 & 0.50 & 4.50 \\
\hline \multicolumn{5}{|l|}{ Housing } \\
\hline Total & & 65.12 & 21.07 & 86.09 \\
\hline
\end{tabular}

Table 2. Part finances.

\begin{tabular}{ccccc}
\hline Component name & Configuration Name & Configuration & Quantity & Total Cost \\
\hline Ball baring & Ball bearing & Default & 2 & 20.00 \\
Spacer & Spacer & Default & 1 & 2.10 \\
Circular section & Top bottom rod & Default & 4 & 10.36 \\
Aluminum (ISO 20111-800) & & & & \\
(ISO 4162-M10X40X26-N) & Hex flange bolt small & Default & 1 pkt & 1.00 \\
(ISO 7412-M16X50-WN) & $\begin{array}{c}\text { Hex structural bolt } \\
\text { grades short }\end{array}$ & Default & $1 \mathrm{pkt}$ & 5.00 \\
Total & & & & 38.46 \\
\hline
\end{tabular}

materials, higher performance efficiencies and lesser costs. Such designs if implemented along the highways, they can produce a larger amount of renewable energy and higher production may help in reducing the unit price.

\section{Conflicts of Interest}

The authors declare no conflicts of interest regarding the publication of this paper.

\section{References}

[1] Bhutta, M.M.A., Hayat, N., Farooq, A.U., Ali, Z., Jamil, S.R. and Hussain, Z. (2012) Vertical Axis wind Turbine-A Review of Various Configurations and Design Techniques. Renewable and Sustainable Energy Reviews, 16, 1926-1939. https://doi.org/10.1016/j.rser.2011.12.004

[2] Jiang, Q., Doyle, J.D., Haack, T., Dvorak, M.J., Archer, C.L. and Jacobson, M.Z. (2008) Exploring Wind Energy Potential off the California Coast. Geophysical Research Letters, 35, L20819. https://doi.org/10.1029/2008GL034674

[3] Rezaeiha, A., Kalkman, I. and Blocken, B. (2017) CFD Simulation of a Vertical Axis Wind Turbine Operating at a Moderate Tip Speed Ratio: Guidelines for Minimum Domain Size and Azimuthal Increment. Renewable Energy, 107, 373-385. 
https://doi.org/10.1016/j.renene.2017.02.006

[4] Jain, P. and Abhishek, A. (2016) Performance Prediction and Fundamental Understanding of Small Scale Vertical Axis Wind Turbine with Variable Amplitude Blade Pitching. Renewable Energy, 97, 97-113.

https://doi.org/10.1016/j.renene.2016.05.056

[5] Wanvik, P.O. (2009) Effects of Road Lighting: An Analysis Based on Dutch Accident Statistics 1987-2006. Accident Analysis \& Prevention, 41, 123-128. https://doi.org/10.1016/j.aap.2008.10.003

[6] Lins, C., Williamson, L.E., Leitner, S. and Teske, S. (2014) The First Decade: 2004-2014: 10 Years of Renewable Energy Progress. Renewable Energy Policy Network for the 21st Century.

[7] Islam, S.M., Nayar, C.V., Abu-Siada, A. and Hasan, M.M. (2018) Power Electronics for Renewable Energy Sources. In: Power Electronics Handbook, 4th Edition, Butterworth-Heinemann, Oxford, 783-827.

https://doi.org/10.1016/B978-0-12-811407-0.00027-1

[8] Olauson, J. and Bergkvist, M. (2015) Modelling the Swedish Wind Power Production Using MERRA Reanalysis Data. Renewable Energy, 76, 717-725.

https://doi.org/10.1016/j.renene.2014.11.085

[9] Balduzzi, F., Bianchini, A., Maleci, R., Ferrara, G. and Ferrari, L. (2016) Critical Issues in the CFD Simulation of Darrieus Wind Turbines. Renewable Energy, 85, 419-435. https://doi.org/10.1016/j.renene.2015.06.048

[10] Hallgren, W., Gunturu, U.B. and Schlosser, A. (2014) The Potential Wind Power Resource in Australia: A New Perspective. PLoS ONE, 9, e99608. https://doi.org/10.1371/journal.pone.0099608

[11] Farthing, S.P. (2009) Vertical Axis Wind Turbine Induced Velocity Vector Theory. Proceedings of the Institution of Mechanical Engineers, Part A: Journal of Power and Energy, 223, 103-114. https://doi.org/10.1243/09576509JPE657

[12] Howell, R., Qin, N., Edwards, J. and Durrani, N. (2010) Wind Tunnel and Numerical Study of a Small Vertical Axis Wind Turbine. Renewable Energy, 35, 412-422. https://doi.org/10.1016/j.renene.2009.07.025

[13] Katsigiannis, Y.A. and Stavrakakis, G.S. (2014) Estimation of Wind Energy Production in Various Sites in Australia for Different Wind Turbine Classes: A Comparative Technical and Economic Assessment. Renewable Energy, 67, 230-236. https://doi.org/10.1016/j.renene.2013.11.051

[14] Polinder, H., Ferreira, J.A., Jensen, B.B., Abrahamsen, A.B., Atallah, K. and McMahon, R.A. (2013) Trends in Wind Turbine Generator Systems. IEEE Journal of Emerging and Selected Topics in Power Electronics, 1, 174-185. https://doi.org/10.1109/JESTPE.2013.2280428

[15] Buchner, A.J., Soria, J., Honnery, D. and Smits, A.J. (2018) Dynamic Stall in Vertical Axis Wind Turbines: Scaling and Topological Considerations. Journal of Fluid Mechanics, 841, 746-766. https://doi.org/10.1017/jfm.2018.112

[16] Rolik, Y. (2004) Some Result of Operating Experience of Wind Turbines in Latvia. Proceeding of International Conference on Wind Energy in the Baltic, Vol. 11, 91-100.

[17] Svitil, K. (2011) Wind-Turbine Placement Produces Tenfold Power Increase, Researchers Say.

[18] Peace, S. (2004) Another Approach to Wind. Mechanical Engineering, 126, 28-31. https://doi.org/10.1115/1.2004-JUN-2 
[19] Buchner, A.J., Lohry, M.W., Martinelli, L., Soria, J. and Smits, A.J. (2015) Dynamic Stall in Vertical Axis Wind Turbines: Comparing Experiments and Computations. Journal of Wind Engineering and Industrial Aerodynamics, 146, 163-171. https://doi.org/10.1016/j.jweia.2015.09.001

[20] Ashwill, T.D., Sutherland, H.J. and Berg, D.E. (2012) A Retrospective of VAWT Technology. Sandia National Laboratories, Albuquerque. https://doi.org/10.2172/1035336

[21] McCroskey, W.J. (1981) The Phenomenon of Dynamic Stall. National Aeronautics and Space Administration Moffett Field Ca Ames Research Center, Mountain View.

[22] Bousman, W.G. (2000) Airfoil Dynamic Stall and Rotorcraft Maneuverability. National Aeronautics and Space Administration Moffett Field CA Ames Research Center, Mountain View.

[23] Rabaglio, L.E. (2015) Análisis aerodinámico del ala delta flexible Extreme.

[24] Akerib, D.S., et al. (2014) First Results from the LUX Dark Matter Experiment at the Sanford Underground Research Facility. Physical Review Letters, 112, Article ID: 091303.

[25] Ramírez, F.J., Honrubia-Escribano, A., Gómez-Lázaro, E. and Pham, D.T. (2017) Combining Feed-In Tariffs and Net-Metering Schemes to Balance Development in Adoption of Photovoltaic Energy: Comparative Economic Assessment and Policy Implications for European Countries. Energy Policy, 102, 440-452.

https://doi.org/10.1016/j.enpol.2016.12.040

[26] Chiras, D. (2010) Wind Power Basics: A Green Energy Guide. New Society Publishers, Gabriola.

[27] Castelli, M.R., Englaro, A. and Benini, E. (2011) The Darrieus Wind Turbine: Proposal for a New Performance Prediction Model Based on CFD. Energy, 36, 4919-4934. https://doi.org/10.1016/j.energy.2011.05.036

[28] Brøndsted, P., Lilholt, H. and Lystrup, A. (2005) Composite Materials for Wind Power Turbine Blades. Annual Review of Materials Research, 35, 505-538. https://doi.org/10.1146/annurev.matsci.35.100303.110641 